Said Another Way

\title{
Seeing the Nursing Profession From a Different Perspective: Nurstry? Why Not!
}

\author{
Huey-Ming Tzeng, PhD, RN, and Chang-Yi Yin, MA
}

Nurses need to identify themselves through a true

revolution. Nurses should be treated as equals with physicians, and nursing as a profession that is capable of independent practice. For this very reason, the term "Nurstry" was invented by the authors to refer to the science and caring art of nursing. Nurstry includes nursing knowledge developed by nurstrists and nurses in the global nursing village. This commentary included sections of discussion as follows: (a) nurses' occupational identity in the time of the Old Testament; (b) nursing profession in the 21st century; (c) smart nurses and the cruel reality in practice; (d) distorted occupational personality; and (e) nurstry as a distinct, professional discipline.
Huey-Ming Tzeng, PhD, RN, is Associate Professor at the Division of Nursing Business and Health Systems, School of Nursing, University of Michigan, Ann Arbor, MI; Chang-Yi Yin, MA, is Professor at the Department of History, Chinese Culture University, Taipei, Taiwan.

$\mathrm{N}$ ursing is well recognized as a profession, a specialized field of study. If this statement is true, nurses desire a specific term to represent nursing as an academic field. If nurses do not have such a self-identity, no one else will recognize nursing as a professional, specialized academic field. A nurses' revolution on ideology is demanded (the way nurses name their own profession and discipline). The first step is to fully recognize the uniqueness of nursing in academia.

Nurses need to identify themselves through a true revolution. Nurses should be treated as equals with physicians, and as a professional that is capable of independent practice. For this very reason, the term "Nurstry" was invented by the authors to refer to the science and caring art of nursing (see Table 1). "Nurstry" includes nursing knowledge developed by "nurstrists" and nurses in the global nursing village. The term "nursing" is used to refer to the profession (e.g., School of Nursing), but this term is not enough to demonstrate the nature of an independent discipline in practice and in academia.

\section{Nurses' Occupational Identity in the Time of the Old Testament}

As indicated in the Holy Bible (Old Testament), nurses and physicians were two independent occupations and held different responsibilities. In the time of the Old Testament (from the seventeenth to the first century BC), nurses practiced independently as wet nurses, dry nurses, midwives, or the ones who cared for the sick. They looked after, fostered, or advised healthy or sick people.

Being midwives and caring for women's health problems were nurses' unique functions. Physicians, 
Said Another Way

Table 1. A Comparison Table for the Newly Invented Terms and the Commonly Adopted Terms Related to Nursing

\section{Newly invented terms}

"Nurstry" as a noun indicates the profession of a nurse (e.g., School of Nurstry), and the science and caring art dealing with the maintenance of health, and the prevention and alleviation of disease.

"Nurstrist" as a noun refers to a scholar or scientist who is involved in the academic research of "nurstry" as his or her primary responsibility.

"Nursing" as an adjective means relating to "nurstry.

\section{Popularly adopted terms}

"Nursing" as a noun indicates the profession of a nurse (e.g., School of Nursing), or the duties of a nurse (Merriam-Webster Online, 2006).

"Nurse" as a noun refers to (a) a person who looks after, fosters, or advises someone, or (b) a person who is skilled or trained in caring for the sick under the supervision of a physician (Merriam-Webster Online, 2006).

"Nurse" as a verb means to care for and wait on a sick person, or to attempt to cure by care and treatment (Merriam-Webster Online, 2006).

\section{Biochemistry and related terms*}

"Biochemistry" as a noun refers to chemistry that deals with the chemical compounds and processes occurring in organisms (Merriam-Webster Online, 2006).

"Biochemist" as a noun.

\begin{abstract}
"Biochemical" as an adjective means (a) to be characterizes by, produced by, or involving chemical reactions in living organisms, or $(\mathrm{b})$ relating to biochemistry (Merriam-Webster Online, 2006).
\end{abstract}

*Biochemistry and related terms were listed here for comparison purposes only.

who were primarily male, could not get close to women and care for female patients. At that time, nurses did not practice under physicians' supervision and did not need to follow physicians' orders for delivering care.

\section{Nursing Profession in the 21st Century}

In practice, physicians are the commanders in patient care, mainly because nurses are required by law to work under the supervision of physicians and by their order. These laws have become an excuse for physicians to "legally" suppress the value of nursing in practice and in academia.
Nurses and physicians do share responsibilities in patient care, and work as a team in practice. Thus, it must be remembered that as early as the time of the Old Testament, nurses practiced independently and did not practice under physicians' supervision for delivering care. Figure 1 depicts the current and expected working relationship between nurses and physicians.

\section{Smart Nurses and the Cruel Reality in Practice}

Head nurses and staff nurses often play an important role in training junior physicians about practical patient care skills and techniques. In patient wards, most residents and medical interns have to count on 
Figure 1. The current and ideal working relationship in clinical practice

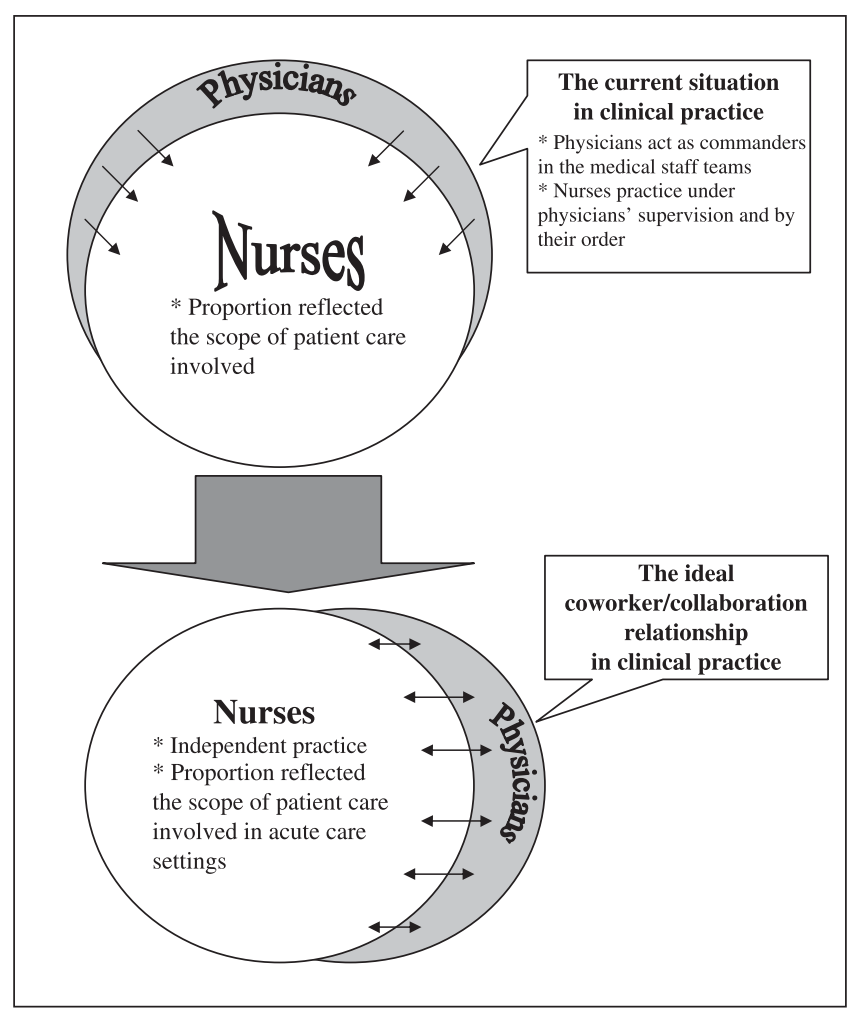

Note: One-way and two-way arrows represent the power structure of the working relationship between nurses and physicians in clinical practice.

nurses for their professional opinions and clinical judgments on medical treatments. Nurses' opinions are based on their professional knowledge and skills in physical assessment, effective communication, observation of patients' needs and condition changes, etc. The fact is that many experienced nurses are way more capable than physicians.

Critical thinking and clinical reasoning are strongly emphasized in nursing education. However, both student nurses and staff nurses quickly learn to be obedient to physicians' orders, as hierarchy and bureaucracy are often emphasized in hospital systems. The nursing practice environment and related legislation are indeed suppressing the development of the nursing profession.

\section{Distorted Occupational Personality}

If the phenomenon described above is true, most nurses are likely to encounter conflicts due to the differences among each individual's personality and characteristics. Each individual's personality, although genetic, can be influenced by (a) an expected occupational personality gained through nursing school education (e.g., being a critical and logical thinker), and (b) a demanded occupational personality formed through clinical practice (e.g., being obedient to physicians and supervisors, being a physician' helper, not having much clinical reasoning). A staff nurse's occupational personality might demonstrate as a human bondage that he or she needs to fit into the culture of the practice environment in order to survive and continuously stay in the nursing profession.

Most of all, the conflicts that arise from differences between the occupational personality cultivated through school education and the demanded occupational personality in practice might have retarded the development of the nursing profession, even in countries with high levels of human development (United Nations Development Programme, 2006).

The occupational personality demanded in clinical nursing practice might not attract high school students to enter the nursing profession. The very same occupational personality may aggravate the difficulties in keeping experienced and outstanding nurses in clinical practice and in academia. The nursing shortage would increase over time for the very same reason.

\section{Nurstry as a Distinct, Professional Discipline}

In academia, nursing and medicine are indeed two disciplines and two divisions of knowledge. Nursing 


\section{Said Another Way}

has its own specific professional characteristics and well-developed master's and PhD programs. Using a keyword search in the Mulford Library database for journals in health sciences (Medical University of Ohio, 2006), we located a total of 341 professional journals related to nurse, nursing, care, or caring. Nursing also has long been identified as an independent academic field in both the social science (SSCI) and science (SCI) divisions of the Journal Citation Reports.

\section{Adopting the term "nurstry" is a step}

forward in promoting the professional image of nurses
Nursing has proved itself to be independent from medicine in the academia. Adopting the term "nurstry" is a step forward in promoting the professional image of nurses and reclaiming their dignity. A revolution on ideology is a must to restore the glory of the nursing profession.

Author contact: tzeng_hueyming@yahoo.com, with a copy to the Editor: nursingforum@gmail.com

\section{References}

Medical University of Ohio. (2006). Mulford library: Instructions to authors in the health sciences. Medical University of Ohio at Toledo. Retrieved March 6, 2006, from http://mulford.mco.edu/ instr.

Merriam-Webster Online (2006). Springfield, MA: Author. Retrieved August 15, 2006, from http://www.m-w.com/cgi-bin/dictionary.

United Nations Development Programme (2006). Human development report 2005. New York: Author. Retrieved August 15, 2006, at http://hdr.undp.org/statistics. 